\title{
Prevalence of chronic kidney disease associated with cardiac and vascular complications in hypertensive patients: a multicenter, nation-wide study in Thailand
}

Rungroj Krittayaphong ${ }^{1 *}$ (D), Ram Rangsin², Bandit Thinkhamrop ${ }^{3}$, Cameron Hurst ${ }^{4}$, Suthee Rattanamongkolgul ${ }^{5}$, Nintita Sripaiboonkij ${ }^{6}$ and Wipaporn Wangworatrakul ${ }^{1}$

\begin{abstract}
Background: Hypertension and chronic kidney disease (CKD) are common conditions and both are major risk factors for cardiovascular events. The objectives were 1) to study the prevalence of CKD in hypertensive patients and 2) to study the association of CKD with cardiac and vascular complications in a multicenter, nation-wide fashion.

Methods: This cross-sectional study evaluated patients aged 20 years or older who were diagnosed with hypertension and who had been treated for at least 12 months at 831 public hospitals in Thailand during the 2012 study period. Outcome measurements included calculated glomerular filtration rate (GFR) and cardiac and vascular complications that included coronary artery disease, stroke, peripheral arterial disease, heart failure, and atrial fibrillation. Multivariable modeling was conducted to determine independent factors associated with increased risk of cardiac and vascular complications.

Results: A total of 28770 patients were enrolled. Average age was 62.8 years and $37 \%$ were male. Prevalence of CKD stage 3 and 4-5 was 33.2 and 4.3\%, respectively. Prevalence of cardiac and vascular complications was 10. $5 \%$ (5\% having coronary artery disease, 3.9\% stroke, 1.7\% heart failure, and 1.2\% atrial fibrillation). CKD was an independent risk factor associated with each of the complications and overall cardiac and vascular complications with an adjusted Odds ratio of 1.4 for CKD stage 3 and 1.9 for CKD stage 4-5.
\end{abstract}

Conclusion: Prevalence of CKD stage 3-5 in hypertensive population was 37.5\%. CKD is an independent risk factor for adverse cardiac and vascular outcome.

Keywords: Chronic kidney disease, Hypertension, Cardiac, Vascular, Complication

\section{Background}

Chronic kidney disease (CKD) is a leading cause of morbidity and mortality among patients around the world [1]. Prevalence of CKD has been estimated to be 8-16\% worldwide [1]. Diabetes and hypertension are major risk factors for the development of CKD [1,2]. Hypertension doubles the risk of CKD in the general population and the risk ratio has been reported to be as

\footnotetext{
* Correspondence: rungroj.kri@mahidol.ac.th

'Division of Cardiology, Department of Medicine, Siriraj Hospital, Mahidol University, 2 Wanglang Road, Bangkoknoi, Bangkok 10700, Thailand

Full list of author information is available at the end of the article
}

high as 3.9 in older population [3]. Control of hypertension can reduce the risk of developing CKD [2].

CKD is classified into 5 stages; CKD stages 1 and 2 have a glomerular filtration rate (GFR) of higher than $60 \mathrm{ml} / \mathrm{min}$. The GFRs of CKD stages 3, 4 and 5 are 30-60, $15-30$ and $<15 \mathrm{ml} / \mathrm{min}$ respectively [4]. Prevalence of the different stages of CKD in Thailand was reported in 2 major studies namely the National Health Exam Survey (NHES) [5] and the Thai Screening and Early Evaluation of Kidney Disease (Thai SEEK) study [6]. The NHES study involved 3117 subjects with the average age of 33.6 years; $22.5 \%$ of subjects had hypertension [5]. Prevalence of CKD stages 3, 4, and 5 from the NHES studies was 8.1, 
0.2 , and $0.15 \%$, respectively. The Thai SEEK study enrolled 3459 subjects with mean age 46.2 years, $16.5 \%$ of patients had a history of hypertension. The Thai SEEK study found prevalence of CKD stages 1-2 and stage 3-4 of 8.9 and $8.6 \%$, respectively [6]. However, there are limited data on the prevalence of CKD in hypertensive population is Asian countries.

Recent practice guidelines for cardiovascular prevention regarded CKD as a major risk factor for cardiovascular event [7]. CKD stages $4-5$ increases risk of cardiovascular event with an Odds ratio of 4-50 [8]. Even in patients with cardiac disease, severity of CKD has been shown to be a better predictor of cardiovascular events than left ventricular ejection fraction $[9,10]$. Prevalence of CKD among patients admitted with acute myocardial infarction was $30-42 \%$ [11]. Patient with CKD had an increased risk of cardiovascular event 2-7 times higher than those without CKD $[8,11]$. There was little data on the association of CKD and cardiovascular outcome in Asian population [12]. It has been reported that patients with CKD are normally more likely to receive treatment that does not closely follow generally accepted practice guidelines than treatment received by patients that do not have CKD [11].

The primary objective of this study was to determine the association between CKD and cardiac and vascular complications in hypertensive patients. The secondary objectives were to: 1) study the prevalence of CKD in hypertensive patients; and 2) study the prevalence of cardiac and vascular complications in patients with hypertension.

\section{Methods}

\section{Study population}

This cross-sectional survey was conducted in Thailand in 2012 to evaluate nation-wide standard of care and treatment outcome among hypertensive patients who were treated at public Thailand Ministry of Public Health $(\mathrm{MoPH})$ hospitals in Thailand and private clinics in Bangkok that participate in the Thailand National Health Security Office (NHSO)'s program. Patients were enrolled if they were diagnosed with hypertension, aged 20 years or older and received regular treatment in the participating hospital or clinic for the past 12 months. All patients were recruited from the ambulatory unit. We excluded patients participating in clinical trials.

We used a two-stage stratified cluster, proportional to the size sampling technique, to select a nationally and provincially representative sample of patients who were diagnosed as hypertension. In provinces outside of Bangkok, participating hospitals included all public hospitals under the oversight of the Thailand Ministry of Public Health $(\mathrm{MoPH})$. For Bangkok, targeted hospitals included all hospitals and clinics that were participated in the Thailand NHSO program. The sampling started at the first stage of 77 strata at the provincial level. The second stage was at the hospital level in each province. In each province, hospitals were stratified into 5 strata, according to sizes. The largest size was more than 500 beds (regional center hospital) followed by $200-500$ beds (provincial general hospital), 90-120 beds (large community hospital), 30-90 beds (medium community hospital), and 10-30 beds (small community hospital). University hospitals were not included from our study.

This study was approved by the Royal Thai Army Medical Department Ethical Review Board, the Ethical Review Committee for Research in Human Subjects, Thailand Ministry of Public Health. The protocol for this study was also approved by the Institutional Review Boards of all participating hospitals. Written informed consent was obtained from subjects prior to participation. This study was supported by the Thai National Health Security Office (NHSO).

\section{Data collection process}

A total of 831 hospitals under the Thai Universal Coverage Scheme were included. The number of regional hospitals, general hospitals and community hospitals were 25,70 , and 736 respectively. Every regional and general hospital was included. Community hospitals were randomly sampled with $70 \%$ of small-sized hospitals, $20 \%$ of medium-sized hospitals and $10 \%$ of large-size community hospitals. These proportions were based on the proportion of health care provided at various hospitals levels. Patients diagnosed as hypertension were randomly selected according to the proportion that were registered at each hospital. Sample size of the study was derived from the proportion to size model.

To minimize the selection bias, all patients who were randomly selected was invited by the study nurse to participate in this study. Data were retrieved from patient' medical records, which included baseline characteristics, presence of hypertensive complications, and laboratory test results. The study nurse was trained for the process of data collection. The required data were retrieved from medical recorded and entered in the case record form (CRF). Entry of data in the CRF was based on data that were written in the medical record or from the ICD-10 diagnostic code. This applied to diagnosis of hypertension and all other diagnoses. A majority of participating hospitals had medical record in non-electronic format limiting the opportunity of data transfer from hospital electronic database. The case record form was then sent to the central data management or MEDRESNET (Medical Research Network). Data management officers at MEDRESNET manually adjudicated the data and ensured that the data in the case record form complied with the study protocol. In case of doubt the query was sent to study site to verify with the data in the medical record. 


\section{Assessment of renal function}

Glomerular filtration rate (GFR) was calculated by Chronic Kidney Disease Epidemiology Collaboration (CKD-EPI) formula [13, 14]. GFR was classified into 3 groups: $\geq 60 \mathrm{ml} /$ $\mathrm{min} ; 30-59 \mathrm{ml} / \mathrm{min}$ (CKD stage 3 ); and, $<30 \mathrm{ml} / \mathrm{min}$ (CKDs stage 4 or 5$)$.

\section{Other measurements}

The following variables were collected: demographic data; height; weight; body mass index (BMI); cardiovascular risk factors; systolic blood pressure (SBP); diastolic blood pressure (DBP); blood chemistry, including fasting plasma glucose (FPG), lipid profiles, serum creatinine, uric acid, proteinuria from urine analysis; available ECG data and results. The laboratory results were the most recent results within 12 months prior to the consent process.

The data management team is responsible for inquiries to study sites to verify data. By a random selection process, site monitoring was performed in approximately 60 hospitals or $10 \%$ of study sites.

Primary outcome measurements were cardiac and vascular complications, which included coronary artery disease (CAD), stroke, peripheral arterial disease (PAD), heart failure, and atrial fibrillation. CAD was defined as angina pectoris, myocardial infarction, or coronary revascularization. Stroke was defined as cerebral infarction, TIA, unspecified stroke, or hemorrhagic stroke. Vascular complication was defined as CAD, stroke, or PAD. Secondary outcomes were defined as individual components of cardiac and vascular complications.

\section{Statistical analysis}

Continuous data were presented as mean and standard deviation and categorical variables were expressed as number and percentage. Comparisons of continuous data were performed by independent samples $t$-test. Comparisons were performed by chi-square test for categorical data. Continuous data were grouped for Odds ratio analysis, as follows: age $\geq$ or $<65$ years, $\mathrm{SBP} \geq$ or $<$ $140 \mathrm{mmHg}, \mathrm{DBP} \geq$ or $<90 \mathrm{mmHg}, \mathrm{TC} \geq$ or $<200 \mathrm{mg} / \mathrm{dl}$, $\mathrm{TG} \geq$ or $<200 \mathrm{mg} / \mathrm{dl}, \mathrm{HDL}<$ or $\geq 40 \mathrm{mg} / \mathrm{dl}, \mathrm{LDL} \geq$ or $<$ $100 \mathrm{mg} / \mathrm{dl}$, BMI $\geq$ or $<25 \mathrm{~kg} / \mathrm{m} 2$, GFR $<$ or $\geq 60 \mathrm{ml} / \mathrm{min}$, and uric acid $\geq$ or $<7 \mathrm{mg} / \mathrm{dl}$ in male and $\geq$ or $<6 \mathrm{mg} / \mathrm{dl}$ in female. When missing data were detected during data entry, query of raw data was sent to the study site for verification. During data analysis, since the number of patients is large and number of missing data was small in the majority of data as shown in Table 2 (meaning that bivariate analysis represents an available case analysis). For the multivariable models complete-cases analysis and multiple imputation analysis were performed. We have now included a forest plot in the results that indicates the effect on missing data. This plot allows comparison between the estimates and 95\% CI from the complete cases and imputed analysis.

Odds ratio and 95\% confidence interval (CI) for univariate analysis were determined by chi-square test. Multivariate logistic regression analysis (with forward LR) was used to determine the independent factors that are associated with increased risk of cardiac and vascular complications using complete-cases analysis with and without imputation method for the missing data. For imputation method, missing values were imputed by using the means of complete cases with noise added based on the $t$-distribution. CKD was tested to determine whether or not it was an independent risk factor associated with cardiac and vascular complications after adjustment for other baseline characteristics. A $p$-value less than 0.05 was considered statistically significant. All statistical analyses were performed using SPSS Statistics version 20 (SPSS, Inc., Chicago, IL, USA).

\section{Results}

There were a total of 28770 patients; with a mean age $62.8 \pm 11.3$ years; $10650(37.0 \%)$ of our subjects were male. Average GFR was $68.3 \pm 22.8 \mathrm{ml} / \mathrm{min}$; $\geq 60 \mathrm{ml} /$ $\mathrm{min}$ in 17970 (62.5\%), 30-59 $\mathrm{ml} / \mathrm{min}$ in 9553 (33.2\%), and $<30 \mathrm{ml} / \mathrm{min}$ in 1247 patients (4.3\%). Prevalence of CKD stage 3 and stages $4-5$ increased with age (Fig. 1) but appeared to have no gender-related predisposition (CKD stage 3 and stages $4-5$ were 35.3 and $4.3 \%$ in men and $32.0 \%$ and $4.4 \%$ in women). Prevalence of cardiac and vascular complications is shown in Table 1. Overall prevalence was $10.5 \%$ with CAD being the most prevalent condition followed by stroke. Cardiac and vascular complications increased with age and men more than women (Fig. 2). Prevalence of all cardiac and vascular complications appears to increase as the level of GFR declined (Fig. 3).

Baseline characteristics, blood chemistry data, control status of cardiovascular risk factors and prevalence of cardiac and vascular complications in each group are shown in Table 2. Hypertension was well controlled (SBP $<140$ and DBP $<90 \mathrm{mmHg}$ ) in $78.0 \%$. FPG was in good control $(<100 \mathrm{mg} / \mathrm{dL})$ in $47.8 \%$ and control for LDL-cholesterol was good $(<100 \mathrm{mg} / \mathrm{dl})$ in $34.9 \%$. The univariate analysis indicates that increased age, male gender, current smoking, general hospital, low BMI, high SBP, low FPG, low total cholesterol, low triglyceride, high HDL-cholesterol, low LDL-cholesterol, and low levels of GFR are associated with cardiac and vascular complications (all $p<0.05$ ). Odds ratios and a forest plot indicating the association of each of the baseline characteristics with cardiac and vascular complications are shown in Fig. 4. When the selected factors from univariate analysis are mutually adjusted in the multivariable modelling, higher age, male gender, general hospital, low 


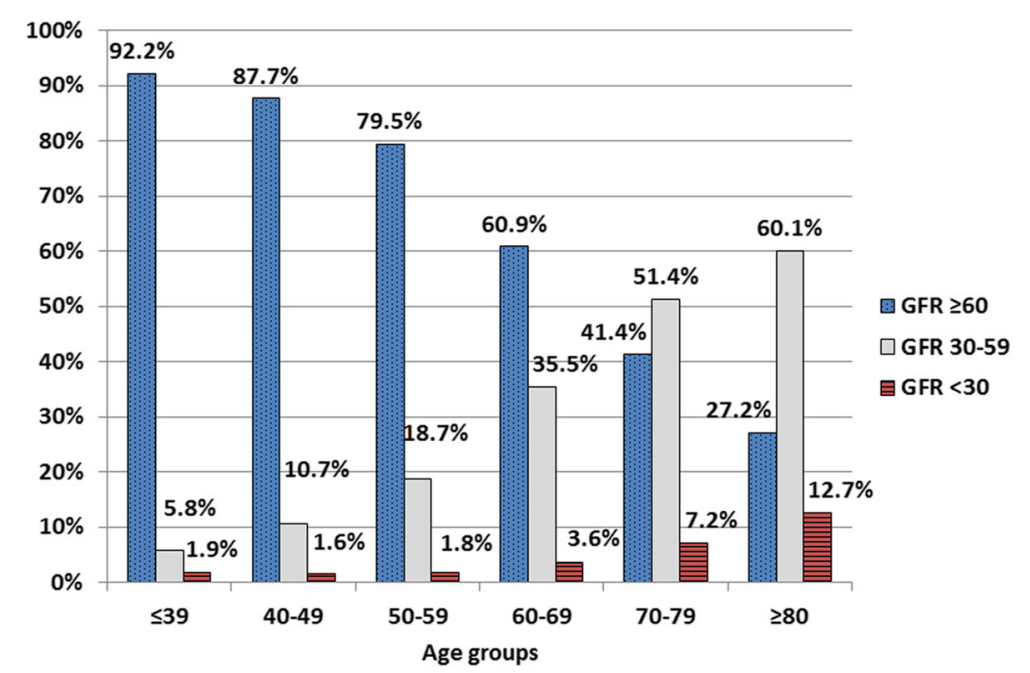

Fig. 1 Prevalence of CKD by age groups

total cholesterol, low triglyceride, high HDL-cholesterol, low LDL-cholesterol, and low levels of GFR are identified as independently associated with cardiac and vascular complications (all $p<0.05$ ). Crude and adjusted Odds ratio of factors that were independently associated with cardiac and vascular complications is shown in Table 3. Forest plot of complete-cases analysis and imputation method is shown in Fig. 5. The direction and significance of the individual effects remains largely unchanged.

Data on proteinuria was available in only 2281 patients (7.9\%). Among patients who had available urine protein data, the results were negative or trace in $874(38.3 \%), 1+$ in 768 (33.7\%), 2+ in 447 (19.6\%), 3+ in 159 (7.0\%), and 4+ in 33 (1.4\%). Since the number of patients with available urine protein data was relatively small, we did not intend to run the primary outcome analysis for proteinuria. However, among patients with urine protein data, proteinuria had an Odds ratio for the association with cardiac and vascular complication of 1.20 (95\% CI $0.92-1.58)$. The numerical association of proteinuria and cardiac and vascular complication was mainly for patients with CKD with the Odds ratio of 1.23 (95\% CI

Table 1 Prevalence of cardiac and vascular complications

\begin{tabular}{ll}
\hline Cardiac and vascular complications & $\begin{array}{l}\text { Number (\%) } \\
\text { (Total N=28770) }\end{array}$ \\
\hline Coronary artery disease & $1442(5.0)$ \\
Stroke & $1118(3.9)$ \\
Peripheral arterial disease & $29(0.1)$ \\
All vascular disease & $2499(8.7)$ \\
Heart failure & $487(1.7)$ \\
Atrial fibrillation & $346(1.2)$ \\
All cardiac and vascular complications & $3031(10.5)$ \\
\hline
\end{tabular}

0.84-1.1.80) compared to those without CKD (Odds ratio $1.00,95 \%$ CI $0.67-1.49$ ).

Multivariable adjusted Odds ratio and 95\% CI for association of levels of GFR with each factor of cardiac and vascular complication are shown in Fig. 6. Results of the analysis showed that the prevalence of each factor of cardiac and vascular complication increased as the level of GFR declined. Multivariable adjusted Odds ratio showed that the level of association between levels of GFR and each of cardiac and vascular complication remained the same with the exception of atrial fibrillation.

\section{Discussion}

The results of this study demonstrated prevalence of CKD stage 3 and $4-5$ in patients with hypertension to be 33.2 and $4.3 \%$, respectively. A decline in GFR in patients with hypertension was independently associated with cardiac and vascular complications which included CAD, stroke, $\mathrm{PAD}$, and heart failure.

Prevalence of CKD has increased over the past 20 years [1] as a result of greater proportion of older people in the population and a corresponding increase in the prevalence of cardiovascular risk factors, such as diabetes and hypertension [1]. Uncontrolled hypertension is a major risk factor for CKD and patients with CKD have an increased risk of hypertension not only from related stiffening of arteries, but also related to volume overload. A world-wide study showed that the prevalence of CKD significantly increases in patients at high risk for cardiovascular disease such as hypertension [15]. Prevalence of left ventricular hypertrophy was as high as $50 \%$ in patients with GFR less than $30 \mathrm{ml} / \mathrm{min}$ [16]. Treatment of hypertension is very effective in the prevention of CKD in patients with hypertension [17]. Blood pressure target in patients 


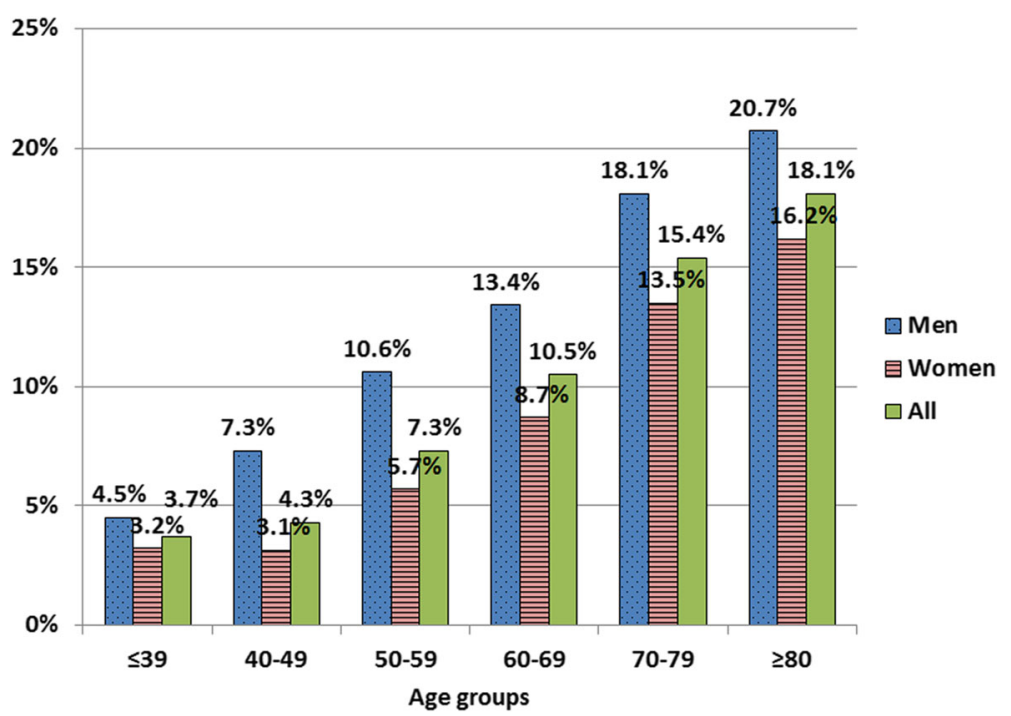

Fig. 2 Prevalence of vascular and cardiac complications in men and women by age groups

with CKD has been proposed to be more stringent than in patients without CKD [18].

Our study showed prevalence of CKD stage 3 and stages 4-5 in patients with hypertension of 33.2 and $4.3 \%$ respectively. Mean age in our study population was 62.8 years. Previous study in healthy Thai individuals from the NHES study showed prevalence of CKD stage 3 and stages $4-5$ of 8.1 and $0.35 \%$, respectively [5]. Data from the Thai SEEK study reported prevalence of CKD stages 3 and 4 of 7.5 and $1.1 \%$, respectively [6]. The difference between our study and other studies mentioned here is mainly the health status of the study population. We studied patients with hypertension while previous studies focused on healthy populations. Prevalence of CKD stage $3-5$ in patients with hypertension was reported to be approximately 23\% [19]. However, our estimate of the prevalence of CKD in the hypertensive population was considerably higher. Although previous studies and our study enrolled patients from primary care centers, the hospitals involved in our study included larger 'regional hospitals'. This difference may have partly influenced the difference in CKD prevalence between our study and previous studies, with a slightly higher mean age of our study population also potentially playing a role.

Patients with CKD stage 3B or 4 (GFR 30-44 and $15-29 \mathrm{ml} / \mathrm{min}$, respectively) had a decreased life expectancy of 17 and 25 years, respectively [20] in comparison with a decrease in life expectancy of 8 years in diabetes [21] and 3 years for hypertension [22]. CKD has recently been identified as a major risk factor for cardiovascular events $[7,8]$.

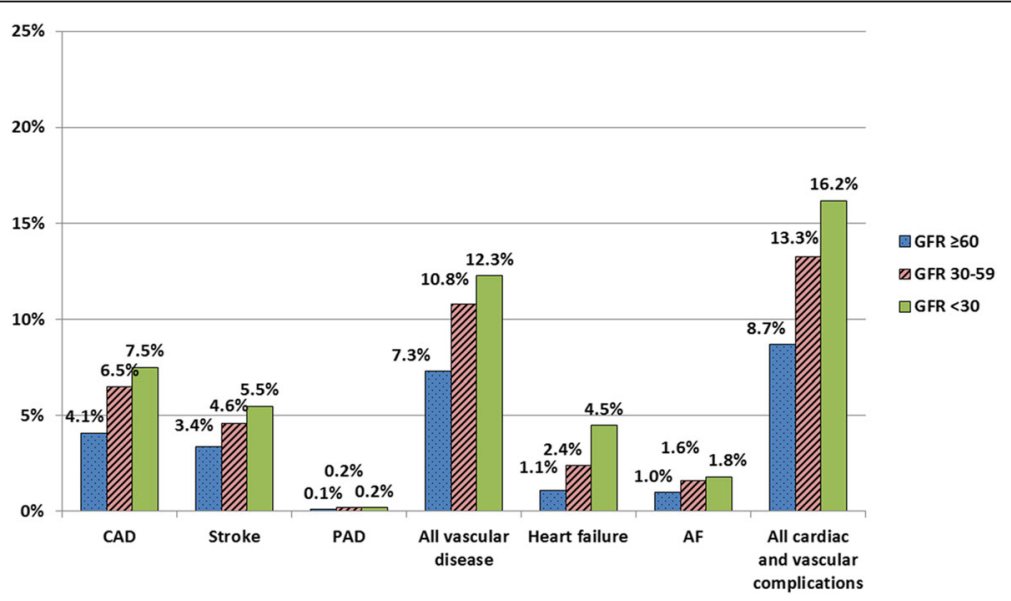

Fig. 3 Prevalence of each type of vascular and cardiac complication by GFR groups 
Table 2 Patient baseline characteristics and prevalence of cardiac and vascular complications (total $N=28770$ )

\begin{tabular}{|c|c|c|c|}
\hline Baseline variables & $n$ & Mean \pm SD/number (\%) & Prevalence of cardiac and vascular complications (\%) \\
\hline Age (years) & 28770 & $62.8 \pm 11.3$ & \\
\hline Gender & 28770 & & \\
\hline Male & & $10650(37.0)$ & 13.8 \\
\hline Female & & $18120(63.0)$ & 8.6 \\
\hline Current smoker & 28770 & & \\
\hline Yes & & $2322(8.1)$ & 14.7 \\
\hline No & & $26448(91.9)$ & 10.2 \\
\hline Type of hospital & 27662 & & \\
\hline General & & $9023(32.6)$ & 13.8 \\
\hline Community & & $18639(67.4)$ & 9.0 \\
\hline SBP $(\mathrm{mmHg})$ & 28758 & $131.1 \pm 15.4$ & \\
\hline$\geq 140$ & & $5814(20.2)$ & 11.4 \\
\hline$<140$ & & $22914(79.8)$ & 10.3 \\
\hline $\mathrm{DBP}(\mathrm{mmHg})$ & 28754 & $75.8 \pm 10.5$ & \\
\hline$\geq 90$ & & $1690(5.9)$ & 10.7 \\
\hline$<90$ & & 27066 (94.1) & 10.5 \\
\hline $\mathrm{FPG}(\mathrm{mg} / \mathrm{dL})$ & 25077 & $107.3 \pm 31.0$ & \\
\hline$\geq 100$ & & 13088 (52.2) & 10.0 \\
\hline$<100$ & & $11989(47.8)$ & 10.9 \\
\hline $\mathrm{TC}(\mathrm{mg} / \mathrm{dL})$ & 25228 & $193.8 \pm 43.3$ & \\
\hline$\geq 200$ & & $10263(40.7)$ & 7.9 \\
\hline$<200$ & & 14965 (59.3) & 11.9 \\
\hline TG (mg/dL) & 26502 & $154.5 \pm 86.8$ & \\
\hline$\geq 200$ & & 5477 (20.7) & 8.6 \\
\hline$<200$ & & 21025 (79.3) & 10.6 \\
\hline $\mathrm{HDL}(\mathrm{mg} / \mathrm{dL})$ & 23933 & $48.8 \pm 13.7$ & \\
\hline$\geq 40$ & & $6060(25.3)$ & 11.8 \\
\hline$<40$ & & $17873(74.7)$ & 9.7 \\
\hline LDL (mg/dL) & 25586 & $115.9 \pm 36.4$ & \\
\hline$\geq 100$ & & $16653(65.1)$ & 8.6 \\
\hline$<100$ & & 8933 (34.9) & 13.1 \\
\hline GFR (ml/min) & 28770 & $68.3 \pm 22.8$ & \\
\hline$\geq 60$ & & $17970(62.5)$ & 8.7 \\
\hline $30-59$ & & 9553 (33.2) & 13.3 \\
\hline$<30$ & & $1247(4.3)$ & 16.2 \\
\hline Uric acid (mg/dL) & 9563 & $6.1 \pm 1.6$ & \\
\hline High & & 3890 (40.7) & 10.5 \\
\hline Normal & & $5673(59.3)$ & 10.1 \\
\hline
\end{tabular}

SD Standard deviation, DM Diabetes mellitus, SBP Systolic blood pressure, DBP Diastolic blood pressure, TC Total cholesterol, TG Triglyceride, HDL High density lipoprotein cholesterol, LDL Low density lipoprotein cholesterol, GFR Glomerular filtration rate by epidemiology collaboration formula

CKD and even proteinuria greatly increase cardiovascular risk and are considered the highest risk group for cardiovascular disease [2] and cardiovascular disease is the major cause of deaths in patients with CKD [23]. A study from Taiwan reported that cardiovascular disease accounted for
$71 \%$ of deaths in patients with CKD, as compared to $22 \%$ in patients with normal kidney function [24]. We demonstrated that CKD in patients with hypertension associated with a substantially increased risk of cardiac and vascular complications including CAD, stroke, PAD, heart failure 


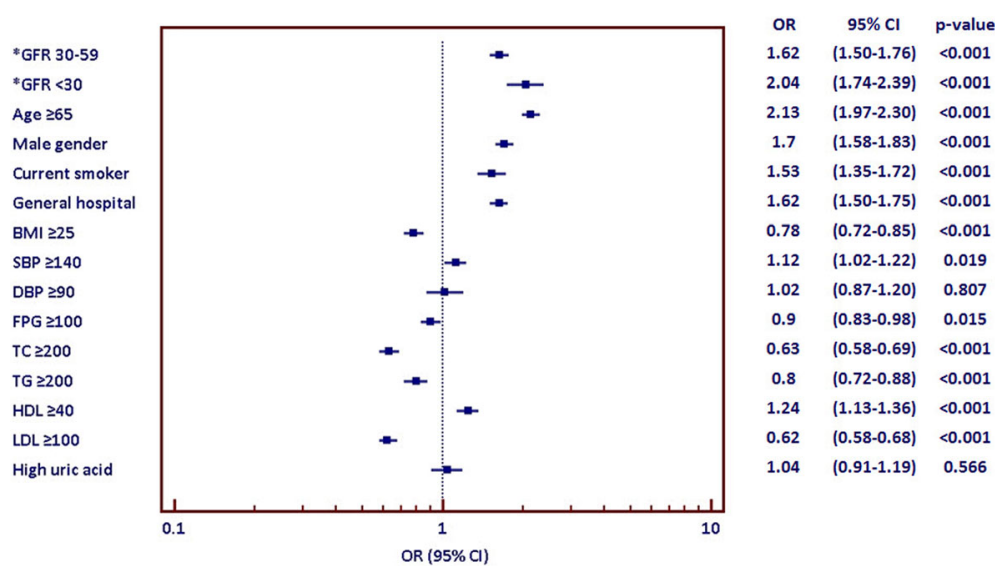

Fig. 4 Bivariate analysis factors affecting cardiac and vascular complications (shown as Odds ratio, 95\% Cl, and forest plot) (*GFR was analyzed by using group with GFR $\geq 60 \mathrm{ml} / \mathrm{min}$ as the reference)

and atrial fibrillation. The adjusted Odds ratio was 1.4 in patients with GFR $30-59 \mathrm{ml} / \mathrm{min}$ and 1.7 for those with GFR below $30 \mathrm{ml} / \mathrm{min}$, in comparison to those with GFR above $60 \mathrm{ml} / \mathrm{min}$. This information is meaningful not only for specialists but also for primary care physicians [25]. We cannot make conclusion on the significance of proteinuria since the proteinuria data was relatively small. However, our data showed increased risk of cardiac and vascular complication of proteinuria in patients with CKD.

Many mechanisms facilitate and contribute to increased cardiovascular risk in CKD. Patients with CKD were commonly found to have concomitant cardiovascular risk factors, such as diabetes and hypertension. Patients with CKD had increased activation of renin-angiotensin and sympathetic response compared to those with normal renal function $[8,26]$. Moreover, patients with CKD had higher levels of inflammatory biomarkers [27] and endothelial dysfunction [28]. In addition to increased risk of cardiovascular disease, patients with CKD, both with and without documented vascular disease, usually received treatment that was less than the treatment practice guidelines, as compared to patients without CKD [11].

Recent report shows a high prevalence of CKD in hypertensive patients and a strong association of CKD and cardiac and vascular complication [15]. This study also shows that more than half of patients with CKD are unaware of this condition. Therefore, these patients have limited opportunity to obtain advice on self-care to slow the progression of CKD and minimize the risk of complications. Registry data from several Asian countries show that a large proportion of CKD patients have a poor control of risk factors especially in those with diabetes [29].

Our study had some limitations. This was a crosssectional study. We did not collect medication data such as anti-hypertensive medications, anti-thrombotic medications, and statins. The nature of our study design cannot prove cause and effect of identified associations. This study was designed for a cross-sectional survey and did not plan for a collection of follow-up data. Therefore

Table 3 Crude and adjusted Odds ratio of factors that were independently associated with cardiac and vascular complications

\begin{tabular}{|c|c|c|c|c|}
\hline Variables & Crude OR (95\% Cl) & $p$-value & Multivariable adjusted OR $(95 \% \mathrm{Cl})$ & $p$-value \\
\hline Age $\geq 65$ years & $2.13(1.97-2.30)$ & $<0.001$ & $1.65(1.48-1.83)$ & $<0.001$ \\
\hline Men & $1.70(1.58-1.83)$ & $<0.001$ & $1.62(1.46-1.80)$ & $<0.001$ \\
\hline General hospital & $1.62(1.50-1.75)$ & $<0.001$ & $1.52(1.37-1.68)$ & $<0.001$ \\
\hline $\mathrm{TC} \geq 200 \mathrm{mg} / \mathrm{dL}$ & $0.63(0.58-0.69)$ & $<0.001$ & $0.82(0.73-0.94)$ & 0.003 \\
\hline $\mathrm{TG} \geq 200 \mathrm{mg} / \mathrm{dL}$ & $0.80(0.72-0.88)$ & $<0.001$ & $0.86(0.75-0.98)$ & 0.023 \\
\hline $\mathrm{HDL} \geq 40 \mathrm{mg} / \mathrm{dL}$ & $1.24(1.13-1.36)$ & $<0.001$ & $1.12(1.00-1.26)$ & 0.045 \\
\hline $\mathrm{LDL} \geq 100 \mathrm{mg} / \mathrm{dL}$ & $0.62(0.58-0.68)$ & $<0.001$ & $0.75(0.66-0.84)$ & $<0.001$ \\
\hline $\mathrm{GFR} \geq 60 \mathrm{ml} / \mathrm{min}$ & reference & $<0.001$ & reference & $<0.001$ \\
\hline $30-59 \mathrm{ml} / \mathrm{min}$ & $1.62(1.50-1.76)$ & & $1.39(1.24-1.55)$ & \\
\hline$<30 \mathrm{ml} / \mathrm{min}$ & $2.04(1.74-2.39)$ & & $1.86(1.50-2.31)$ & \\
\hline
\end{tabular}




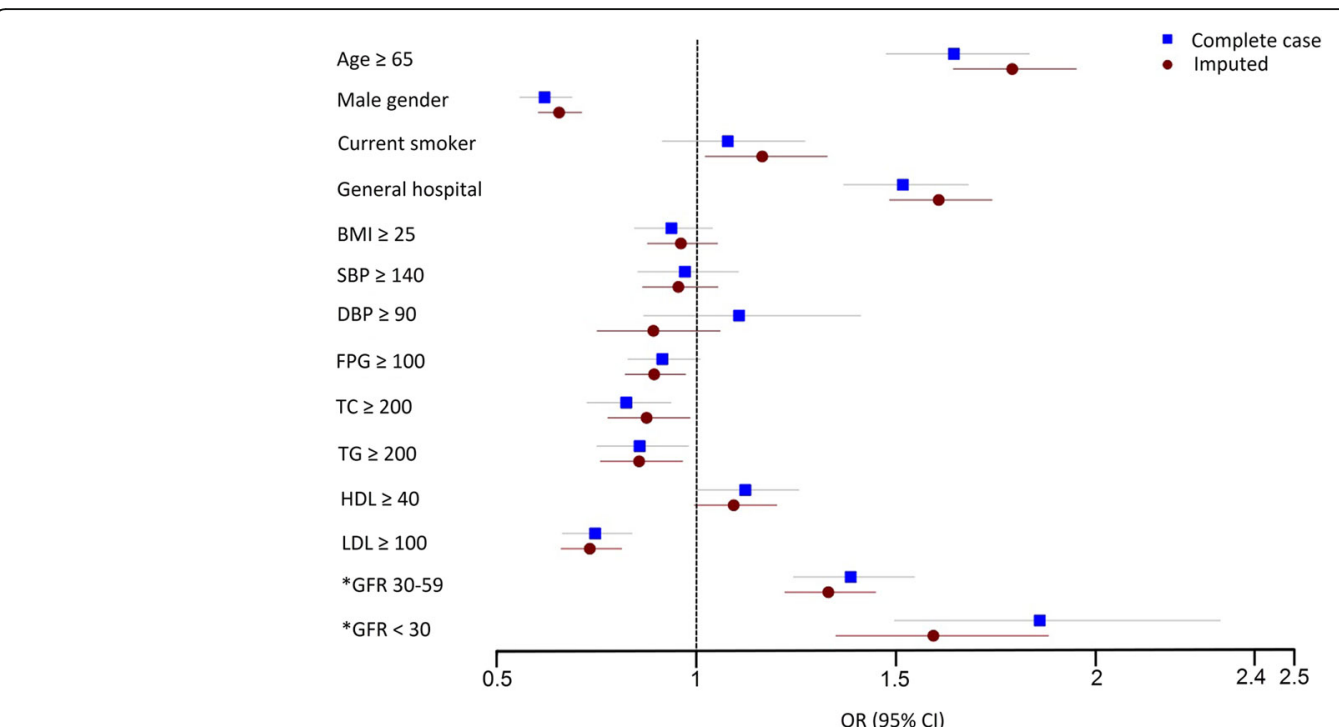

Fig. 5 Forest plot shows Odds ratio and $95 \% \mathrm{Cl}$ of multivariate analysis of factors affecting cardiac and vascular complications from complete case analysis and imputation method ( ${ }^{*}$ GFR was analyzed by using group with GFR $\geq 60 \mathrm{ml} / \mathrm{min}$ as the reference)

we did not collect short or long-term outcome or changes in GFR or proteinuria. In fact, the main purpose of the main study was to determine the rate of blood pressure goal achievement and to feedback to the participating hospitals how good they are for blood pressure control. This study collected data from hospital based samples which may over-estimate the prevalence obtained from community based individuals. All patients were recruited from the ambulatory setting. Therefore the probability of recruiting acute kidney injury (AKI) cases should be very low since AKI cases should be admitted and managed in hospital setting. However, undetected AKI may be missed. The associations between cardiac and vascular complications with low cholesterol, low LDL-cholesterol, low triglyceride, and high HDLcholesterol could be due to the effect of statin use, which would be more commonly found in patients with cardiac and vascular disease. This could make some results of our study difficult to interpret. Methods of creatinine measurement were not the same across all participating centers. Approximately half of the centers used enzymatic method for the measurement of serum creatinine and another half used modified Jaffe method. Enzymatic method was IDMS (isotope dilution-mass spectrometry)-traceable. Although the method that was used in many centers were not IDMS-traceable but all centers had their own laboratory standard quality control system that applied to local practice. Lastly, there

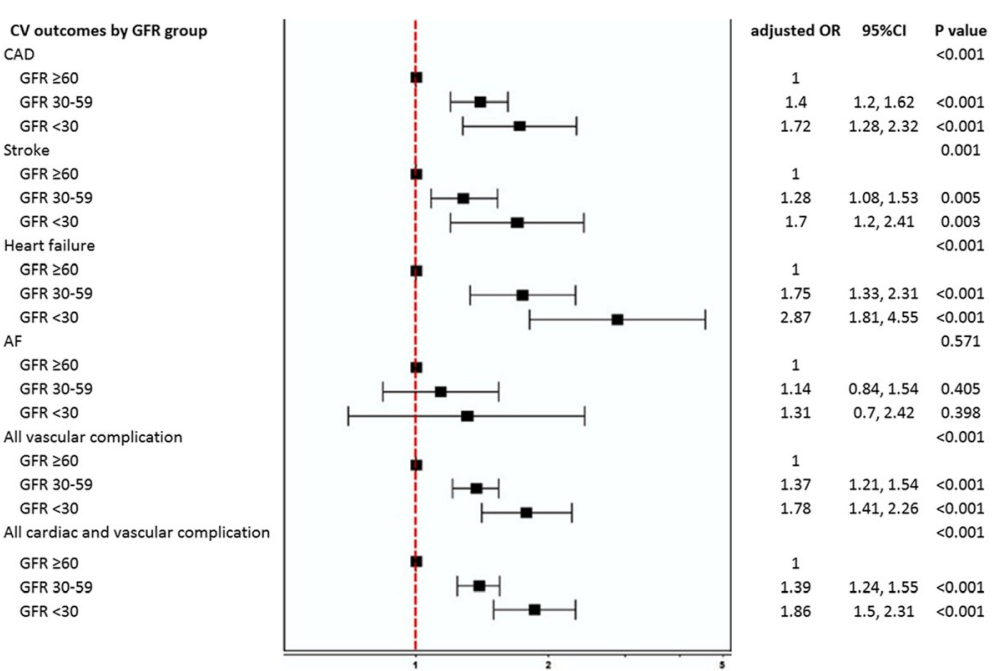

Fig. 6 Multivariable relationships between GFR groups and each type of cardiac and vascular complication 
were some cases with missing data, since it was not mandatory to have every variable entered into the case record form.

\section{Conclusion}

In conclusion, prevalence of CKD in our hypertensive population was $37.5 \%$. CKD is an independent risk factor that is associated with many cardiac and vascular complications. Improved awareness regarding detection and management of CKD is very important, especially for primary care physicians.

\begin{abstract}
Abbreviations
AF: Atrial fibrillation; Cl: Confidence interval; CKD: Chronic kidney disease; CRF: Case record form; DBP: Diastolic blood pressure; DM: Diabetes mellitus, ECG: Electrocardiogram; EPI: Epidemiology collaboration formula; FPG: Fasting plasma glucose; GFR: Glomerular filtration rate; HDL: High density lipoprotein; LDL: Low density lipoprotein; MoPH: Ministry of public health; NHSO: National health security office; PAD: Peripheral arterial disease; SBP: Systolic blood pressure; SD: Standard deviation; TC: Total cholesterol; TG: Triglyceride; TIA: Transient ischemic attack
\end{abstract}

\section{Acknowledgements}

We thank Ahthit Yindeengam for the assistance in data management and illustration.

\section{Funding}

This study was funded by a grant from a Thai National Health Security Office (NHSO).

\section{Availability of data and materials}

The dataset of this article is available in http://www.damus.in.th.

\section{Authors' contributions}

Conception and design (RK, RR and SR), data acquisition, statistical analysis and interpretation of data (BT, CH, NS, and WW), manuscript preparation (RK and $\mathrm{CH}$ ), revising manuscript with important intellectual content (RK, RR, BT, $\mathrm{CH}$, and SR), review of manuscript (RK, RR, BT, CH, SR, NS, and WW). All authors read and approved the final manuscript.

\section{Competing interests}

The authors hereby declare no personal or professional conflicts of interest regarding any aspect of this study.

\section{Consent for publication}

Not applicable.

\section{Ethics approval and consent to participate}

This study was approved by the Royal Thai Army Medical Department Ethical Review Board, the Ethical Review Committee for Research in Human Subjects, Thailand Ministry of Public Health. The protocol for this study was also approved by the Institutional Review Boards of all participating hospitals. Written informed consent was obtained from subjects prior to participation.

\section{Publisher's Note}

Springer Nature remains neutral with regard to jurisdictional claims in published maps and institutional affiliations.

\section{Author details}

'Division of Cardiology, Department of Medicine, Siriraj Hospital, Mahidol University, 2 Wanglang Road, Bangkoknoi, Bangkok 10700, Thailand. ${ }^{2}$ Department of Military and Community Medicine, Phramongkutklao College of Medicine, Bangkok, Thailand. ${ }^{3}$ Faculty of Public Health, Khon Kaen University, Khon Kaen, Thailand. ${ }^{4}$ Faculty of Medicine, Chulalongkorn University, Bangkok, Thailand. ${ }^{5}$ Department of Preventive and Social Medicine, Srinakarinwirot University, Nakornnayok, Thailand. ${ }^{6}$ Cancer Registry Unit, Ramathibodi Hospital, Mahidol University, Bangkok, Thailand.
Received: 15 August 2016 Accepted: 23 March 2017

Published online: 03 April 2017

\section{References}

1. Jha V, Garcia-Garcia G, Iseki K, Li Z, Naicker S, Plattner B, et al. Chronic kidney disease: global dimension and perspectives. Lancet. 2013;382:260-72.

2. Gansevoort RT, Correa-Rotter R, Hemmelgarn BR, Jafar TH, Heerspink HJ, Mann JF, et al. Chronic kidney disease and cardiovascular risk: epidemiology, mechanisms, and prevention. Lancet. 2013;382:339-52.

3. Kearns B, Gallagher H, de Lusignan S. Predicting the prevalence of chronic kidney disease in the English population: a cross-sectional study. BMC Nephrol. 2013;14:49.

4. Vassalotti JA, Stevens LA, Levey AS. Testing for chronic kidney disease: a position statement from the National Kidney Foundation. Am J Kidney Dis. 2007:50:169-80.

5. Ong-Ajyooth L, Vareesangthip K, Khonputsa P, Aekplakorn W. Prevalence of chronic kidney disease in Thai adults: a national health survey. BMC Nephrol. 2009;10:35

6. Ingsathit A, Thakkinstian A, Chaiprasert A, Sangthawan P, Gojaseni P, Kiattisunthorn $K$, et al. Prevalence and risk factors of chronic kidney disease in the Thai adult population: Thai SEEK study. Nephrol Dial Transplant. 2010;25:1567-75.

7. Perk J, De Backer G, Gohlke H, Graham I, Reiner Z, Verschuren M, et al. European Guidelines on cardiovascular disease prevention in clinical practice (version 2012). The Fifth Joint Task Force of the European Society of Cardiology and Other Societies on Cardiovascular Disease Prevention in Clinical Practice (constituted by representatives of nine societies and by invited experts). Eur Heart J. 2012;33:1635-701.

8. Schiffrin EL, Lipman ML, Mann JF. Chronic kidney disease: effects on the cardiovascular system. Circulation. 2007;116:85-97.

9. Hillege HL, Girbes AR, de Kam PJ, Boomsma F, de Zeeuw D, Charlesworth A, et al. Renal function, neurohormonal activation, and survival in patients with chronic heart failure. Circulation. 2000;102:203-10.

10. Hillege HL, Nitsch D, Pfeffer MA, Swedberg K, McMurray JJ, Yusuf S, et al. Renal function as a predictor of outcome in a broad spectrum of patients with heart failure. Circulation. 2006;113:671-8.

11. Fox CS, Muntner P, Chen AY, Alexander KP, Roe MT, Cannon CP, et al. Use of evidence-based therapies in short-term outcomes of ST-segment elevation myocardial infarction and non-ST-segment elevation myocardial infarction in patients with chronic kidney disease: a report from the National Cardiovascular Data Acute Coronary Treatment and Intervention Outcomes Network registry. Circulation. 2010;121:357-65.

12. Hsing SC, Lu KC, Sun CA, Chien WC, Chung CH, Kao SY. The Association of Losartan and Ramipril Therapy With Kidney and Cardiovascular Outcomes in Patients With Chronic Kidney Disease: A Chinese Nation-Wide Cohort Study in Taiwan. Medicine. 2015;94:e1999.

13. Levey AS, Stevens LA, Schmid CH, Zhang YL, Castro 3rd AF, Feldman HI, et al. A new equation to estimate glomerular filtration rate. Ann Intern Med. 2009;150:604-12.

14. Stevens LA, Schmid CH, Greene T, Zhang YL, Beck GJ, Froissart M, et al. Comparative performance of the CKD Epidemiology Collaboration (CKD-EPI) and the Modification of Diet in Renal Disease (MDRD) Study equations for estimating GFR levels above 60 mL/min/1.73 m2. Am J Kidney Dis. 2010;56:486-95.

15. Ene-lordache B, Perico N, Bikbov B, Carminati S, Remuzzi A, Perna A, et al. Chronic kidney disease and cardiovascular risk in six regions of the world (ISN-KDDC): a cross-sectional study. Lancet Glob Health. 2016;4:e307-19.

16. Levin A, Singer J, Thompson CR, Ross H, Lewis M. Prevalent left ventricular hypertrophy in the predialysis population: identifying opportunities for intervention. Am J Kidney Dis. 1996;27:347-54.

17. Kokubo Y, Nakamura S, Okamura T, Yoshimasa Y, Makino H, Watanabe M, et al. Relationship between blood pressure category and incidence of stroke and myocardial infarction in an urban Japanese population with and without chronic kidney disease: the Suita Study. Stroke. 2009;40:2674-9.

18. Upadhyay A, Earley A, Haynes SM, Uhlig K. Systematic review: blood pressure target in chronic kidney disease and proteinuria as an effect modifier. Ann Intern Med. 2011;154:541-8.

19. Gomez P, Ruilope LM, Barrios V, Navarro J, Prieto MA, Gonzalez O, et al. Prevalence of renal insufficiency in individuals with hypertension and obesity/overweight: the FATH study. J Am Soc Nephrol. 2006;17:S194-200.

20. Hemmelgarn BR, Clement F, Manns BJ, Klarenbach S, James MT, Ravani P, et al. Overview of the Alberta Kidney Disease Network. BMC Nephrol. 2009;10:30. 
21. Franco OH, Steyerberg EW, Hu FB, Mackenbach J, Nusselder W. Associations of diabetes mellitus with total life expectancy and life expectancy with and without cardiovascular disease. Arch Intern Med. 2007;167:1145-51.

22. Loukine L, Waters C, Choi BC, Ellison J. Health-Adjusted Life Expectancy among Canadian Adults with and without Hypertension. Cardiol Res Pract. 2011;2011:612968.

23. Turin TC, Tonelli M, Manns BJ, Ravani P, Ahmed SB, Hemmelgarn BR. Chronic kidney disease and life expectancy. Nephrol Dial Transplant. 2012;27:3182-6.

24. Wen CP, Cheng TY, Tsai MK, Chang YC, Chan HT, Tsai SP, et al. All-cause mortality attributable to chronic kidney disease: a prospective cohort study based on 462293 adults in Taiwan. Lancet. 2008:371:2173-82.

25. Singh TK, Arya V, Navaratnarajah N. Chronic Kidney Disease and Cardiovascular Disease: A Focus on Primary Care. Cardiovascular \& hematological disorders drug targets. 2014;14:212-8.

26. Tomey MI, Winston JA. Cardiovascular Pathophysiology in Chronic Kidney Disease: Opportunities to Transition from Disease to Health. Ann Global Health. 2014;80:69-76.

27. Krane V, Wanner C. Statins, inflammation and kidney disease. Nat Rev Nephrol. 2011;7:385-97.

28. Ochodnicky P, Henning RH, van Dokkum RP, de Zeeuw D. Microalbuminuria and endothelial dysfunction: emerging targets for primary prevention of end-organ damage. J Cardiovasc Pharmacol. 2006;47 Suppl 2:S151-62. discussion S72-6.

29. Luk AO, Li X, Zhang Y, Guo X, Jia W, Li W, et al. Quality of care in patients with diabetic kidney disease in Asia: The Joint Asia Diabetes Evaluation (JADE) Registry. Diabetic medicine : a journal of the British Diabetic Association. 2016;33:1230-9.

\section{Submit your next manuscript to BioMed Central and we will help you at every step:}

- We accept pre-submission inquiries

- Our selector tool helps you to find the most relevant journal

- We provide round the clock customer support

- Convenient online submission

- Thorough peer review

- Inclusion in PubMed and all major indexing services

- Maximum visibility for your research

Submit your manuscript at www.biomedcentral.com/submit

C) Biomed Central 